\title{
PROVERBES ET SAVOIRS INFORMELS AU BÉNIN (AFRIQUE DE L'OUEST) ${ }^{1}$
}

\author{
Eric Adja ${ }^{2}$
}

\section{Introduction}

Jusqu'à présent, les travaux consacrés aux proverbes de l'Afrique ont rarement dépassé le stade de la collecte, de la transcription et de la traduction-interprétation, dans le cadre des préoccupations d'ordre ethnographique ou littéraire dont a fait l'objet la tradition orale africaine. La révision théorique qu'a connu la communication, ainsi que l'apparition de notions comme la coénonciation et la coconstruction du sens permettent de dépasser le décodage pur et simple du vouloir-dire qui a longtemps caractérisé l'étude des genres de la littérature orale africaine (contes, légendes, devinettes, etc.). En effet, une analyse en termes de contextes d'emploi et de travail cognitif opéré tant au niveau de l'émission que de la réception peut permettre d'éclairer d'un jour nouveau ces modalités du déploiement de l'acte de parole dont on sait l'importance dans les cultures

1 Je youdrais exprimer ici ma gratitude envers Jean-Pierre Meunier, Philippe Verhaegen, Jean Lohisse et les autres enseignants du Département de communication de l'Université catholique de Louvain, dont les cours ont inspiré la présente réflexion. J'en assume cependant les qualités et les défauts.

2 Universités de Genève et Paris 7, Laboratoire Langage, Langues et Culture d'Afrique Noire, LLACAN (UMR 7594) du CNRS. 
africaines. C'est dans cette perspective que nous proposons une relecture des proverbes du fongbe, langue parlée par les Fons du SudBénin, en Afrique de l'Ouest. Nos observations sur le terrain nous ont persuadé que les proverbes de cette langue peuvent être analysés non seulement comme discours sociaux (impliquant des dispositifs d'énonciation), mais également comme "technologies de l'intelligence" (selon l'expression de J. Goody), mettant en cuvre des dispositifs d'acquisition et de communication des savoirs.

Cette recherche s'inscrit donc dans le cadre théorique de la sémiotique cognitive, une approche qui cherche à préciser l'articulation entre les signes externes (langage verbal et images matérielles notamment) et les signes internes (images et modèles mentaux). Selon ce courant théorique, les signes externes ne sont pas de simples moyens de communication des signes internes, car le fonctionnement cognitif se trouve co-déterminé par la médiation sémiotique. Il s'agit donc d'explorer l'hypothèse d'une relation de circularité et de corrélation entre le sociosémiotique et le psychologique et de "préciser comment les signes externes élaborés dans l'activité socio-discursive rétroagissent sur les formes de l'imagerie mentale, leur différenciation et leur organisation"1.

Dans la première partie, nous présenterons une caractéristique originale du proverbe fon, son interactivité. En effet, la possibilité d'une énonciation à double entrée sous forme de question-réponse permettant au locuteur de laisser le soin à son interlocuteur de deviner ou d'imaginer la suite du proverbe selon l'environnement mutuel partagé, permet une sorte de coconstruction ou de coénonciation au terme de laquelle la signification est construite "en temps réel" au cours de l'échange interlocutoire. Dans la deuxième partie, nous discuterons du fonctionnement cognitif des proverbes, dont l'usage se fonde sur la culture commune des interlocuteurs, laquelle instaure des représentations du monde et des modèles mentaux plus ou moins complexes. Ces modèles mettent en œuvre des constructions faisant intervenir divers éléments de la nature, de l'histoire, de la vie quotidienne, ce qui fait des proverbes de véritables encyclopédies orales et informelles qui s'enrichissent au fil des interactions et tissent la toile d'un savoir pratique et global.

1 J.-P. MEUnier, "Connaître par l'image", Recherches en communication, $\mathrm{n}^{\circ} 10$, Louvain-la-Neuve, 1999, pp. 35-75. 


\section{La communication par les proverbes}

Loin d'être un phénomène révolu, l'usage des proverbes dans les interactions verbales est encore d'actualité au Bénin (et en Afrique en général), où, vu le faible taux de scolarisation, plus de $3 / 4$ de la population acquiert et communique ses savoirs dans les langues locales, en dehors des salles de classe. Cependant la pratique des proverbes entre parfois en confrontation avec l'enseignement formel (dispensé en français) où des formules comme "soyez brefs", "venons-en au fait", "évitez les précautions oratoires", "appelez un chat un chat"... laissent perplexes des élèves à cheval entre deux cultures, l'une influencée par l'oralité et l'autre par l'écriture. Les Fons utilisent les proverbes dans l'interaction verbale, afin de rendre celle-ci vivante, d'exprimer une pensée abstraite en termes d'images, pour la rendre plus accessible à l'interlocuteur. Il s'agit donc de combiner raison, imagination et humour, dans une sorte de communication visant à la communion avec l'interlocuteur. On entrevoit déjà à quel point l'apprentissage et la pratique des proverbes résultent de l'immersion sociale du sujet et se déroulent dans un contexte de négociation permanente du sens, ce qui demande à l'individu une constante ouverture au monde et aux autres.

\subsection{Le dispositif d'énonciation}

Il existe deux types de proverbes chez les Fons, le "proverbe à un énoncé" et le "proverbe à deux énoncés". Le premier type permet de communiquer un message à travers une phrase, une interrogation, une interjection, une observation de la nature. Il correspond à la définition que donne du proverbe le Larousse (2000), un "court énoncé exprimant un conseil populaire, une vérité de bon sens ou d'expérience..." comme c'est le cas dans les exemples (1) et (2).

(1) Gbe o to dema we. E xo nyi disi o e no xo yi amyon: "la vie est comme un roseau au bord de l'eau, qui penche tantôt d'un côté, tantôt de l'autre" (pour évoquer les vicissitudes, les "hauts et les bas" de l'existence). 
(2) Hun de dyo o wefo ton no dyo: "Quand le rythme du tambour change, les pas du danseur changent aussi" (nécessité de s'adapter aux changements).

Le second type, plus courant et plus original est celui qui retiendra notre attention. En effet, le proverbe à deux énoncés fonctionne de façon interactive, selon une modalité d'action-réaction. Ce type de proverbe donne lieu à une véritable mise en scène au cours de la conversation. Comme dans le proverbe (3), un locuteur A cite le premier énoncé, laissant le soin à son interlocuteur $\mathrm{B}$, censé connaître le proverbe, de le compléter explicitement ou mentalement. Deux possibilités se présentent alors :

- Ou l'interlocuteur connaît le proverbe et dans ce cas, réplique le deuxième énoncé, ce qui a pour conséquence de clôturer la session du proverbe et de "faire avancer le débat" entre les interlocuteurs.

- Ou, ne connaissant pas le proverbe, pose la question “qu'est-ce qu'il dit ?". Dans ce cas, la balle est renvoyée à l'émetteur qui restitue le second énoncé et poursuit son discours.

(3) A : Anuwanumono na nu nukuno: "Un fou donne l'aumône à un aveugle?"

B : Bo ne e do ? : "Et que dit-il ?"

A : Hun mede gbe le balawe hu ce : "Il y a donc plus malheureux que moi !"

Une "session d'échange de proverbes" au cours de l'interaction est assez brève (moins d'une minute), mais elle relance considérablement l'échange interlocutoire et instaure un climat de sympathie et de détente du discours, bref de communion. On peut découper cette session en 4 états (schéma) :

- État initial (E0): Ouverture de la session (émission du premier énoncé du proverbe par $\mathrm{A}$ ).

- État intermédiaire (E1) : Réception et traitement par B.

- État de rétroaction (E2): Retour de $\mathrm{B}$ à $\mathrm{A}$ (restitution du second énoncé ou question).

- État final (E3) : Clôture conjointe de la session par A et B (mise en relation du proverbe avec le contexte de l'interaction) et poursuite de la discussion. 


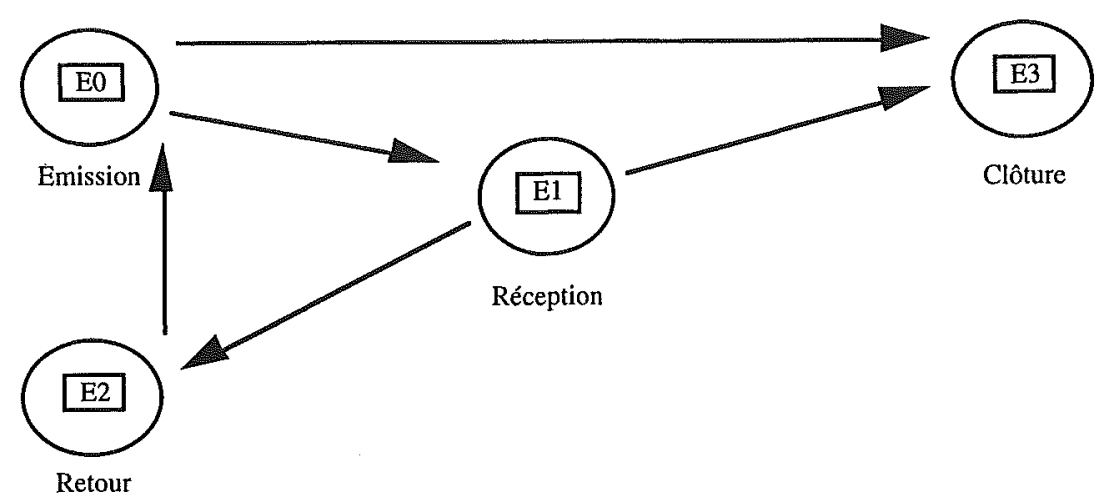

Schéma : une session d'échange par proverbe

Dans la suite du texte, nous simplifierons la citation des proverbes en représentant uniquement les deux états E0 (émission) et $\mathrm{E} 2$ (rétroaction), respectivement par A et $\mathrm{B}$.

\subsection{Interactivité et coopération inférentielle dans les proverbes fons}

Comme nous venons de le voir, au niveau de l'émission, chaque proverbe est le fruit d'un travail individuel et collectif. Travail individuel, car, quand bien même les proverbes sont censés être connus de tous, leur polysémie autorise l'individu à les employer dans des contextes différents, à en élargir ou à en restreindre le sens, à créer de nouvelles associations métaphoriques en fonction de son imagination. Travail collectif, car le proverbe ne prend sens que dans un contexte précis, dans un mouvement de cocréation entre interlocuteurs. L'initiateur du premier énoncé veillera donc à ne pas transformer son proverbe en devinette ou en énigme, mais à le rendre suffisamment accessible pour que le but visé soit atteint, celui d'expliciter sa pensée. Le proverbe est donc donné à titre d'un nœud sémiotique à défaire, mais un nœud partiellement défait par les indices contenus dans le premier énoncé. A ce niveau, on peut noter une coopération entre les interlocuteurs, en fonction de l'environnement mutuel partagé. Nous ne sommes pas loin de la notion de coopération de Grice, devenue une notion importante dans la compréhension des processus de communication. Selon Grice en effet : 
Nous pourrions ainsi formuler en première approximation un principe général qu'on s'attendra à voir respecté par tous les participants : que votre contribution conversationnelle corresponde à ce qui est exigé de vous, au stade atteint par celle-ci, par le but ou la direction acceptée de l'échange parlé dans lequel vous êtes engagé. Ce qu'on pourrait appeler principe de coopération ${ }^{1}$.

$\mathrm{Au}$ niveau de la réception et de la rétroaction, le proverbe nécessite une activité essentielle d'inférence. Pour ce faire, la communication par le biais d'un proverbe est garantie par le respect du principe de pertinence (Sperber et Wilson) ${ }^{2}$ selon lequel une information pertinente pour un individu est une information qui modifie et améliore la représentation globale que cet individu a du monde. Ainsi, partant du premier énoncé du proverbe considéré comme indice, et supposant que l'émetteur respecte le principe de pertinence, le récepteur infère toutes les hypothèses possibles sur l'histoire, les us et coutumes des Fons, l'actualité, les symbolismes des animaux, des végétaux, des astres, les divinités... en d'autres termes, le monde comme environnement mutuel partagé. L'activité inférentielle d'un destinataire $B$ qui essaie de répondre à la question : "qu'est-ce que l'émetteur A veut me dire à travers ce proverbe, sur la base de notre monde mutuellement partagé ?", ou qui, ne connaissant pas le proverbe, demande à A : "que dit ce proverbe ?" implique une décentration et une intériorisation du point de vue de son interlocuteur. Le destinataire B devient alors locuteur dans l'interaction en étant celui qui pose la question. Il se produit une inversion de pôles interchangeables et une restitution de l'intra-individuel à l'interindividuel. Le dénouement du proverbe ainsi est compris comme le résultat d'un travail commun. Le proverbe fon apparaît ici donc comme un instrument d'ouverture à la communication (émission individuelle du premier énoncé) et de communication de l'ouverture (restitution collective du second énoncé). Dans un tel contexte, l'individu ne saurait se concevoir comme une entité fermée par rapport aux autres.

On rejoint ici un point de vue de la psychosociologie d'inspiration systémique, qui situe le point de départ de la communication dans l'interaction elle-même et non dans le moi individuel. Une interaction avec le monde d'une part, et avec l'autre d'autre part qui donne

1 P.H. GRICE, "Logique et conversation", Communications, $\mathrm{n}^{\circ}$ 30, 1979, p. 61.

2 D. SPERber et D. WILson, La pertinence, Paris, Éd. de Minuit, 1989, p. 242. 
naissance aux sujets individualisés. Basée sur des principes de coopération et de pertinence l'utilisation des proverbes du fongbe ne consiste pas seulement en la communication d'un message à décoder, mais en la modification et en l'élargissement de l'environnement cognitif mutuel des interlocuteurs. La compréhension d'un proverbe suppose donc un travail de reconstruction d'un modèle mental en relation avec celui de l'émetteur. Ce travail se fonde sur une certaine ouverture à l'intention de l'interlocuteur. C'est cette activité inférentielle qui confère au proverbe fon une portée cognitive, qui reste à explorer.

\section{La valeur cognitive des proverbes fons}

Tout est métaphore dans le savoir parémiologique des Fons, un savoir qui ne vise pas à l'acquisition pure et simple de connaissances désincarnées par rapport au monde et à l'environnement immédiats (comme c'est souvent le cas pour l'enseignement formel hérité de la colonisation en Afrique), mais plutôt à la sagesse, ce savoir-faire éthique spontané qui donne à penser la cognition comme activité concrète de tout l'organisme.

\subsection{Histoire, raison et imagination}

Les proverbes fons mettent en scène un registre large et varié de personnages utilisés comme support du savoir à communiquer. Les personnages mis en scène sont des humains de toutes conditions (un homme riche, un fou, un lépreux, une commerçante...), mais aussi des animaux, des végétaux, des divinités, des astres, etc. Les personnages humains par exemple apparaissent lorsqu'il s'agit de proverbes avec des références historiques, géographiques, politiques, religieuses, etc. Ces proverbes véhiculent à la fois une idée précise (en rapport avec l'objet de l'interaction au cours de laquelle ils interviennent) et d'autre part un savoir plus large et plus global qui dépasse le temps et l'espace d'émission du proverbe.

Ainsi dans le proverbe (4), il est question de la période de l'esclavage ( $\mathrm{XVII}^{\mathrm{e}}-\mathrm{XIX}^{\mathrm{e}}$ siècles) dont on sait que le Bénin, ancienne côte des esclaves fut l'un des hauts lieux de la traite négrière. Ce proverbe est aussi le témoin du contact à cette époque et de la 
collaboration entre les autochtones et les Européens, les Portugais notamment. Il s'agit ici d'un pan de l'histoire du Bénin, enseignée dans les écoles, mais diffusée de façon informelle par les proverbes, en un raccourci saisissant enrobé d'humour; pourtant au second niveau le même proverbe est employé dans un contexte où le locuteur décline sa responsabilité dans une situation donnée :

(4) A : E gbo ba do xo kanumo do aguda ma xwe wu : "L'on bastonne un esclave pour ne pas avoir été acheté par les Portugais".

B : Hwe ce kawe a : "Est-ce ma faute ?" ou "Suis-je responsable de cette mévente?" (s'exclame l'esclave).

Dans le proverbe (5) il est question du personnage d'Agaja qui fut roi du Danxome de 1708 à 1732 , une époque où les rois détenaient le droit de vie ou de mort sur leurs sujets. Ce proverbe constitue une autre incursion dans l'histoire du Bénin. N'a-t-il pas aussi a posteriori une valeur politique? Ne s'agirait-il pas aussi d'une critique du pouvoir absolu des souverains, d'une revendication détournée du droit à la vie et à la liberté ? Car en effet le proverbe en question sert à dénoncer tout harcèlement, toute fatigue oppressante dont on est l'objet :

(5) A : Nyade xwe ku bo Agaja do wen ni : "Au moment de son exécution, un quidam se voit remettre par le roi Agadja, un message pour les ancêtres".

B : Se we te gboje mi a: "Quoi, serai-je privé de repos à tout jamais ?" (pour devoir jouer les coursiers à la solde du roi, même dans l'au-delà...).

Dans le proverbe (6), il est question du savoir religieux, du contact des populations locales avec la religion chrétienne, même si le proverbe est employé pour notifier à un individu sa part de responsabilité dans les problèmes auxquels il se trouve confronté.

(6) A : Anuwanumono we mon jesu kike do akusu ji : "Un fou aperçoit le Christ sur une croix" (un crucifix).

B : Nyae, hwi lo o, nude jen a wa : "Mon gars, il n'est pas sûr que tu sois tout à fait innocent dans cette histoire..." (dit le fou à l'adresse du Christ...). 
Le grand nombre d'espèces animales (une cinquantaine, sur un corpus d'environ un millier de proverbes dont la moitié ont pour personnages des animaux) et végétales fait des proverbes des véritables traités de zoologie et de botanique. Du crabe à l'éléphant, en passant par la libellule, le rat, le lion ..., le locuteur du fongbe apprend à travers les proverbes, les noms et caractéristiques de divers mammifères, quadrupèdes, palmipèdes, etc., selon un fonctionnement quasi mnémotechnique.

\subsection{Du savoir informel au savoir éthique}

Chez les Fons, il n'existe pas d'école pour apprendre les proverbes. On les acquiert et les utilise dans le contexte de l'immersion sociale. L'acquisition des proverbes ne concerne pas des savoirs désincarnés par rapport à la réalité. Il s'agit au contraire de savoirs qui englobent la totalité de l'environnement des interlocuteurs. Il s'agit à la fois d'un savoir objectif, rationnel (puisque le proverbe survient au cours de l'interaction pour argumenter et préciser une idée, une proposition), mais aussi d'un savoir faisant appel à l'imagination, à la créativité et à l'ouverture au point de vue d'autrui. Le moi n'est plus tout à fait le centre unique de production du sens, mais il intègre la présence des autres. Il s'agit donc d'un savoir éthique, au sens où l'emploie Varela, c'est-à-dire un savoir qui mène à "la prise de conscience progressive et directe de la virtualité du moi"'. S'inspirant des traditions orientales et bouddhistes, cet auteur pense qu'on peut définir l'apprentissage notamment éthique comme la reconnaissance de la vacuité fondamentale du moi, ce qui crée chez l'individu un sentiment d'ouverture, de sympathie, d'inclusion, de compassion, une aptitude à la décentration qui est tout l'opposé de l'égocentrisme.

La notion de "savoir informel" serait-elle donc plus proche de celle de "savoir éthique" de Varela ? Nous sommes porté à le penser ; car les savoirs que communiquent les proverbes des Fons du Bénin, à l'instar du proverbe (7) sont des savoirs qui réconcilient imagination et raison.

1 F. J. VARELA, Quel savoir pour l'éthique ? Action, sagesse et cognition, Paris, Éd. de La Découverte, 1996. 
(7) A : Anuwanumono fleko nu Legba do hoja : "Un fou arrache la tête à la divinité nommée Legba" (une statue représentant une divinité).

B : A kpon Ahoko kpede hun, no kpon hoja lo kpede : "Tu dois regarder dans un sens comme dans l'autre" (faire preuve d'objectivité).

Le personnage du fou est très présent dans les proverbes fons. Le fou y incarne la déviance relative de l'imagination par rapport à une norme rationalisée ; d'ailleurs, il n'est pas rejeté (en règle générale, au Bénin et en Afrique, le fou est bien intégré dans sa société). Dans les proverbes fon, il est même réhabilité, car c'est lui qui tient un discours rationnel et invite à l'objectivité, à l'impartialité dans le raisonnement. En tournant la tête de la divinité au risque de lui tordre le cou, et en l'invitant à regarder dans un sens comme dans l'autre, à considérer la thèse et l'antithèse de toutes situations, le fou abolit tout argument d'autorité et nous invite à emprunter d'autres chemins que ceux de notre confortable conformisme. Ce proverbe en appelle à la fois à l'imagination et à la raison et invite à une prise en compte de l'imagination dans la conception de la cognition humaine. Ce qui nous rapproche du point de vue de Johnson ${ }^{1}$ qui plaide pour la reconnaissance du rôle joué par l'imagination dans toute signification, dans notre appréhension de la réalité et dans notre façon de donner sens à notre expérience.

\section{Conclusion}

S'il est vrai que la préoccupation fondamentale du proverbe fon est la compréhension de l'autre dans le contexte de l'interaction, il s'agit toujours d'une compréhension où l'imagination et la raison vont de paire. En faisant appel à une compréhension plus ou moins subjective par l'imagination, le proverbe fon intègre ces deux dimensions et ne considère pas l'homme comme séparé de son environnement.

Notre exploration des proverbes fons nous permet de risquer une définition, ou tout au moins une caractérisation des savoirs informels qui se distinguent à notre avis des savoirs formels, à deux niveaux :

1 M. Johnson, The body in the mind, Chicago, The University of Chicago Press, 1987. 
- au niveau du dispositif d'énonciation caractérisé par une plus grande ouverture aux notions de coopération et de pertinence ;

- au niveau cognitif, par la notion de savoir éthique et incarné, accordant une plus grande place à l'imagination.

Contrastant ainsi avec un certain solipsisme présent dans le discours unilatéral, impersonnel et froidement "rationalisé" de l'enseignement formel actuel dispensé en français au Bénin, les proverbes fons nous invitent à ce mariage entre science et sagesse, mais pas en tant qu'un corpus de normes morales, mais plutôt en tant qu'une technique de communication de savoirs englobant l'environnement proche et lointain des protagonistes de l'interaction linguistique. Savoir, dans ce sens, c'est savoir faire face à soi-même, à l'autre et à l'environnement mutuellement partagé. Dans ce sens aussi, comme l'écrivait Malraux dans les Noyers de l'Altenburg, celui qui sait, ce "n'est pas seulement celui à qui les livres sont nécessaires, mais tout homme dont une idée, si élémentaire soit-elle, engage et ordonne la vie". 